\title{
THE NOTION OF APPROXIMATE EIGENVALUES APPLIED TO AN INTEGRAL EQUATION OF LASER THEORY*
}

$\mathrm{BY}$

\author{
H. J. LANIDAU
}

Bell Laboratories, Murray Hill

\begin{abstract}
The integral operator with kernel $(i \eta / \pi)^{1 / 2} \exp \left[-i \eta(x-y)^{2}\right]$ on the interval $|x|,|y| \leq 1$ serves to model the behavior of a class of lasers. Although the kernel is simple, it is not Hermitian; this presents a major obstacle to a theoretical understanding of the equation-indeed, even the existence of eigenvalues is difficult to prove. We here introduce a definition of approximate eigenvalues and eigenfunctions, and argue that these will model the physical problem equally well. We then show that, for $\eta$ sufficiently large, each point $\lambda$ with $|\lambda|=1$ is an approximate eigenvalue, and that the number of mutually orthogonal approximate eigenfunctions corresponding to $\lambda$ grows faster than any constant multiple of $\sqrt{ } \eta$. This confirms a conjecture of $J$. A. Cochran and E. W. Hinds, supported by numerical evidence. In physical terms, it shows that for large Fresnel number the laser cannot be expected to settle to a single mode.
\end{abstract}

Introduction and conclusions. For a laser with rectangular plane-parallel reflectors which are mirror images of each other, the integral operator

$$
A_{\eta} f \equiv(i \eta / \pi)^{1 / 2} \int_{-1}^{1} \exp \left[-i \eta(x-y)^{2}\right] f(y) d y, \quad|x| \leq 1,
$$

with $\eta$ a real parameter, describes the way in which the field distribution $f(y)$ is transformed by the laser [1]. Interest focuses on those field distributions which reproduce themselves, to within some complex constant $\lambda$, on a single passage through the laser. These are called "modes", and $1-|\lambda|^{2}$ is termed the energy loss of the mode.

If a field distribution has the form $f(y)=\sum_{1}{ }^{M} a_{k} \varphi_{k}(y)$, with each $\varphi_{k}$ a mode, then $L$ passages through the laser transform $f(y)$ into

$$
f_{L}(y)=\sum_{1}^{M} a_{k} \lambda_{k}{ }^{L} \varphi_{k}(y)=\lambda_{1}{ }^{L}\left[a_{1} \varphi_{1}(y)+\sum_{2}^{M} a_{k}\left(\frac{\lambda_{k}}{\lambda_{1}}\right)^{L} \varphi_{k}(y)\right] \text {. }
$$

Thus if $\left|\lambda_{1}\right|$ is the largest of the $\left\{\left|\lambda_{k}\right|\right\}$ (arranged in decreasing order), the effect of many iterations is to attenuate the second component in the above decomposition relative to the first, with the result that the field in the laser converges to $\varphi_{1}(y)$, the mode of lowest energy loss. This desirable phenomenon is sometimes referred to as "the natural selection of modes". Its efficacy clearly depends on the size of the ratio $\left|\lambda_{2}\right| /\left|\lambda_{1}\right|$, which determines the rapidity with which the remaining modes attenuate; indeed, if $\left|\lambda_{1}\right|=$ $\left|\lambda_{2}\right|>\left|\lambda_{3}\right|$, the field distribution behaves like $a_{1} \lambda_{1}{ }^{L} \varphi_{1}(y)+a_{2} \lambda_{2}{ }^{L} \varphi_{2}(y)$ and so does not

\footnotetext{
* Received March 22, 1976.
} 
settle to a single shape at all. The natural question hereby raised concerning the distribution of large $\left|\lambda_{k}\right|$ has not previously been answered, however, for despite its rather tantalizing simplicity, little is known about $A_{\eta}$. A comprehensive account of the information available appears in [1], where it is conjectured, with the support of numerical evidence, that the $\left\{\lambda_{k}\right\}$ lie on a spiral $S_{\eta}$ which approaches the unit circumference as the Fresnel number $\eta \rightarrow \infty$.

If we take literally our definition of mode, and our mathematical representation of the laser, then a mode corresponds to an eigenfunction: $\lambda_{k} \varphi_{k}=A_{\eta} \varphi_{k}$. Since the kernel of $A_{\eta}$ is square-integrable over the region $|x|,|y| \leq 1, A_{\eta}$ is a compact operator. It follows that each nonzero eigenvalue occurs with finite multiplicity, and that the only possible limit point of the eigenvalues is zero [2, p. 167]. However, since $A_{\eta}$ is not Hermitian, general theory largely stops here. The eigenfunctions can be very sparse when they exist at all, and, should there be too few to span the totality of field distributions, information about them need not shed much light on the action of the operator. ${ }^{1}$ These considerations apply to the laser equation, for although a hard-won result shows that eigenfunctions do exist, nothing is known about their completeness, so that their value for an understanding of $A_{\eta}$ is open to doubt.

We can remove some of these conceptual difficulties by recognizing that, whether in the laboratory or in a computation, we necessarily work within a certain error, which can be very small but is always non-zero. Thus if our mathematical models are to reflect the actual conditions we face, they cannot be forced to make distinctions which we are incapable of making, such as between quantities which are strictly zero and those which are exceedingly small. In this light, we see that what should really concern us in modeling is not exclusively the true eigenvalues and eigenfunctions, but rather the whole class of approximate ones, which we define as follows, using energy as norm:

$$
\|\varphi\|^{2}=\int_{-\infty}^{\infty}|\varphi(x)|^{2} d x
$$

Definition: Given $\epsilon>0, \lambda$ is an $\epsilon$-approximate eigenvalue of an operator $A$ if for some function $\varphi_{\lambda}$ of unit norm $\left\|A \varphi_{\lambda}-\lambda \varphi_{\lambda}\right\|<\epsilon$; we call $\varphi_{\lambda}$ an $\epsilon$-approximate eigenfunction corresponding to $\lambda$.

In this definition, we think of $\epsilon^{2}$ as representing the limit of accuracy (in measuring percentages of energy), so that the $\epsilon$-approximate eigenvalues and eigenfunctions are what would be indistinguishable from true ones under the prevailing conditions, and we seek results which do not depend on the actual value of $\epsilon$ in force; thus our definitions have physical meaning. If $\lambda$ is an $\epsilon$-approximate eigenvalue so, according to the definition, is every $\mu$ sufficiently close to $\lambda$, so that we cannot count the approximate eigenvalues directly as points; we can, however, count them by the number of corresponding approximate eigenfunctions which are mutually orthogonal.

If $A$ possesses a complete orthonormal set of true eigenfunctions, then by expanding an $\epsilon$-approximate eigenfunction $\varphi_{\lambda}$ in this basis we can see that the $\epsilon$-approximate eigenvalue $\lambda$ lies within $\epsilon$ of a true eigenvalue, and for $\epsilon$ sufficiently small $\varphi_{\lambda}$ itself lies close to a true eigenfunction. Hence in all such situations, and in particular for integral operators with Hermitian kernels, our present notions of $\epsilon$-approximate eigenvalues and eigen-

${ }^{1}$ A simple example which illustrates this problem is given by the operator which shifts a sequence $\left(a_{1}, a_{2}, \ldots, a_{k}\right)$ into $\left(a_{2}, a_{3}, \ldots, a_{k}, 0\right)$; here there is only one eigenvector, $(1,0, \ldots, 0)$, with eigenvalue 0 . 
functions neighbor those of true ones, as intuitively they should. The distinction between them arises, and plays a crucial role, in passing to non-Hermitian equations where, as we have seen, true eigenvalues do not in general describe the operator adequately; on the other hand, at least for operators of convolution type, systems of mutually orthogonal $\epsilon$-approximate eigenfunctions are easily constructed, and the behavior of $\epsilon$-approximate eigenvalues, as well as their role in explaining the action of the operator, carry over smoothly from the Hermitian case [3]. When we remember that, for $\epsilon$ sufficiently small, we cannot distinguish operationally between true and $\epsilon$-approximate, the possibility arises that in certain non-Hermitian contexts it is the second notion that should replace the first at the center of the stage, even for purposes of theory.

Returning to our original concern with mode selection, suppose that the initial field distribution has the form $f(y)=\sum_{1}{ }^{M} a_{i} \varphi_{j}(y)+e_{0}(y),|y| \leq 1$, in which $\left\{\varphi_{i}\right\}$ are orthonormal $\epsilon$-approximate eigenfunctions of $A_{\eta}$ and $e_{0}(y)$ is a small error, $\left\|e_{0}\right\|<\epsilon$; then

$$
\begin{aligned}
A_{\eta} f=\sum_{1}^{M} a_{i} A_{\eta} \varphi_{i}+A_{\eta} e_{n} & =\sum_{1}^{M} a_{j} \lambda_{j} \varphi_{i}+\sum_{1}^{M} a_{i}\left(A_{\eta} \varphi_{j}-\lambda_{i} \varphi_{j}\right)+A_{\eta} e_{0} \\
& =\sum_{1}^{M} a_{i} \lambda_{j} \varphi_{i}+e_{1} .
\end{aligned}
$$

To estimate $e_{1}$ we invoke the triangle and Schwarz inequalities, together with the fact, evident from (4) below, that $A_{\eta}$ does not increase the norm; we obtain

$$
\begin{aligned}
\left\|e_{1}\right\| & \leq\left\|A_{\eta} e_{0}\right\|+\sum_{1}^{M}\left|a_{i}\right|\left\|A_{\eta} \varphi_{i}-\lambda_{i} \varphi_{i}\right\| \\
& \leq\left\|e_{0}\right\|+\left(\sum_{1}^{M}\left\|A_{\eta} \varphi_{i}-\lambda_{i} \varphi_{i}\right\|^{2} \sum_{1}^{M}\left|a_{i}\right|^{2}\right)^{1 / 2} \\
& \leq\left\|e_{0}\right\|+\left(|| f-e_{0} \|^{2} M \epsilon^{2}\right)^{1 / 2} \leq C \epsilon,
\end{aligned}
$$

where the constant $C$ depends only on the energy of $f$ and the number of terms in the expansion. Consequently, after $L$ passages through the laser, the distribution $f(y)$ becomes

$$
\sum_{i}^{M} a_{i} \lambda_{i}{ }^{L} \varphi_{i}(y)+e_{L}(y),
$$

with $\left\|e_{L}\right\| \leq C^{L} \epsilon$. Thus if we can take $\epsilon$ sufficiently small-that is, if the approximate eigenfunctions look sufficiently like true ones-they will yield the same conclusions regarding mode selection, at least up to the number $L$ of iterations being envisaged. Thus, as earlier, we are led to consider the $\epsilon$-approximate eigenvalues of large modulus, a problem we will examine for $\eta \rightarrow \infty$. We note from the outset that, since $A_{\eta}$ decreases the norm, the modulus of $\epsilon$-approximate eigenvalues is necessarily bounded above by 1 , for if $|\lambda|>1,\left\|A_{\eta} \varphi-\lambda_{\varphi}\right\| \geq\left\|\lambda_{\varphi}\right\|-\left\|A_{\eta} \varphi\right\| \geq|\lambda|-1$, so that $\lambda$ cannot serve as an $\epsilon$-approximate eigenvalue for any $\epsilon<|\lambda|-1$.

When $k(x)$ is a Hermitian symmetric $(k(-x)=\overline{k(x)})$ integrable function whose Fourier transform $K(u)$ is integrable, the eigenvalues of the finite convolution equation

$$
\lambda f(x)=\rho /(2 \pi)^{1 / 2} \int_{-1}^{1} k[\rho(x-y)] f(y) d y, \quad|x| \leq 1,
$$

are well described by a theorem of Szegö [4], which asserts that, as $\rho \rightarrow \infty$, they behave 
like samples of $K(u)$ taken at integer multiples of $\pi / \rho$. Since this conclusion depends in an essential way on the Hermitian nature of $k$, it cannot be employed here. We will instead base ourselves on a recent result [3] which provides, for approximate eigenvalues, a certain extension of Szegö's theorem to the non-Hermitian case. However, since that discussion likewise requires integrability of the kernel, we will modify it, giving a simple self-contained construction. Our conclusions are that, given $\epsilon>0$, each point $\gamma$ on the unit circumference is an $\epsilon$-approximate eigenvalue of $A_{\eta}$ as soon as $\eta$ is sufficiently large, that the number of mutually orthogonal $\epsilon$-approximate eigenfunctions corresponding to $\gamma$ grows faster than any constant multiple of $\sqrt{ } \eta$, and that if $\gamma_{1} \neq \gamma_{2}$ these associated eigenspaces can be chosen to be orthogonal. This accords well with the calculations of [1] already mentioned, where even for relatively low values of $\eta$, the (approximate) eigenvalues of large modulus spiral close to the unit circumference. In view of the earlier discussion, we see that we cannot expect the mode selection process to be effective for large values of the Fresnel number $\eta$.

Proof. We proceed to a proof of the following theorem.

Theorem: Let $A_{\eta} f \equiv(i \eta / \pi)^{1 / 2} \int_{-1}^{1} \exp \left(-i \eta(x-y)^{2}\right) f(y) d y,|x| \leq 1$. Let there be given real positive $C$ and $\epsilon$, and a complex number $\gamma$ with $|\gamma|=1$. Then for all sufficiently large values of the real parameter $\eta$, there are at least $C \sqrt{ } \eta$ functions $\left\{\varphi_{k}\right\}$ orthonormal on $|x| \leq 1$ satisfying $\int_{-1}{ }^{1}\left|A_{\eta} \varphi(x)-\gamma \varphi(x)\right|^{2} d x<\epsilon^{2}$. If $\left|\gamma_{1}\right|=\left|\gamma_{2}\right|=1$ but $\gamma_{1} \neq \gamma_{2}$, these collections of $\epsilon$-approximate eigenfunctions corresponding to $\gamma_{1}$ and $\gamma_{2}$ can be chosen to be mutually orthogonal.

Proof: Let $T$ represent the Fourier transform

$$
T^{\prime} g \equiv \frac{1}{(2 \pi)^{1 / 2}} \int_{\ldots \infty}^{\infty} g(x) \exp (-i u x) d x \equiv C_{i}(u),
$$

with inverse transform

$$
g(x)=T^{-1} G(u)=\frac{1}{(2 \pi)^{1 / 2}} \int_{-\infty}^{\infty} G(u) \operatorname{cxp}(i u x) d u .
$$

We know that these operations preserve energy:

$$
\|T h\|^{2}=\|h\|^{2}
$$

for each $h$. Now with $\varphi(x)$ given on $|x| \leq 1$ and normalized so that $\int_{-1}{ }^{1}|\varphi(x)|^{2} d x=1$, let us extend $\varphi(x)$ as 0 for $|x|>1$, denoting the resulting function by $\varphi^{*}(x)$ :

$$
\begin{aligned}
\varphi^{*}(x) & =\varphi(x), & & |x| \leq 1 \\
& =0, & & |x|>1 .
\end{aligned}
$$

This extension allows us to simplify the form of some of the quantities of interest, since now we can write $A_{\eta} \varphi$ as a convolution

$$
A_{\eta \varphi}=(i \eta / \pi)^{1 / 2} \int_{-\infty}^{\infty} \exp \left(-i \eta(x-y)^{2}\right) \varphi^{*}(y) d y,
$$

and produce the bound

$$
\int_{-1}^{1}\left|A_{\eta} \varphi(x)-\gamma \varphi(x)\right|^{2} d x \leq \int_{-\infty}^{\infty}\left|A_{\eta} \varphi(x)-\gamma \varphi^{*}(x)\right|^{2} d x .
$$


We next appeal to the fact that the formula for the Fourier transform of a convolution can be applied to $A_{\eta} \varphi$, even though the kernel is not integrable [5, p. 58, p. 178]; denoting $T \varphi^{*}$ by $\Phi(u)$ we thus obtain

$$
T A_{\eta} \varphi=\exp \left(i u^{2} / 4 \eta\right) \Phi(u) .
$$

Finally, combining (2) with the right-hand side of (3), we find

$$
\begin{aligned}
\int_{-1}^{1}\left|A_{\eta} \varphi(x)-\gamma \varphi(x)\right|^{2} d x & \leq \int_{-\infty}^{\infty}\left|T\left(A_{\eta} \varphi-\gamma \varphi^{*}\right)\right|^{2} d u \\
& =\int_{-\infty}^{\infty}\left|\operatorname{xxp}\left(i u^{2} / 4 \eta\right)-\gamma\right|^{2}|\Phi(u)|^{2} d u .
\end{aligned}
$$

Our object is to construct functions $\varphi^{*}(x)$ which make the right-hand side of (5) small. To this end, let $S_{\eta}$ be a set of the $u$-axis on which

$$
\left|\exp \left(i u^{2} / 4 \eta\right)-\gamma\right|^{2}<\epsilon^{2} / 2,
$$

and suppose that we can find a function $\Phi(u)$, the Fourier transform of some $\varphi(x)$ of unit energy vanishing in $|x|>1$, which is well-concentrated on $S_{\eta}$, in the sense that

$$
\int_{s_{n}}|\Phi(u)|^{2} d u \geq 1-\epsilon^{2} / 8 .
$$

Then by (2),

$$
\int_{u S_{1}}|\Phi(u)|^{2} d u \leq \epsilon^{2} / 8,
$$

so that

$$
\begin{array}{rl}
\int_{-\infty}^{\infty}\left|\exp \left(i u^{2} / 4 \eta\right)-\gamma\right|^{2}|\Phi(u)|^{2} & d u \\
& =\int_{u \in S_{\eta}}+\int_{u \epsilon s_{\eta}} \leq \frac{\epsilon^{2}}{2} \int_{u \epsilon \aleph_{\eta}}|\Phi(u)|^{2} d u+4 \int_{u \uparrow, s_{\eta}}|\Phi(u)|^{2} d u \\
& \leq \epsilon^{2} / 2+4\left(\epsilon^{2} / 8\right)=\epsilon^{2},
\end{array}
$$

as desired. We conclude that any orthonormal collection $\left\{\varphi_{k}\right\}$ of functions supported on $|x| \leq 1$ whose Fourier transforms $\left\{\Phi_{k}(u)\right\}$ are each well-concentrated on $S_{\eta}$ (in the sense of $(7)$ ) yields $\epsilon$-approximate eigenfunctions of $A_{\eta}$ corresponding to the $\epsilon$-approximate eigenvalue $\gamma$. We next give a simple estimate of the number of such functions.

The set defined by (6) is composed of those points $u$ for which $\left|u^{2} / 4 \eta-\arg \gamma+2 k \pi\right|$ $\leq 2 \sin ^{-1}(\epsilon / 2 \sqrt{ } 2)$, with some $k=0, \pm 1, \pm 2, \cdots$ hence can be expressed as the set of points of the form $\sqrt{ } \eta V$ where $V$, independent of $\eta$, is the union of the intervals $\mid v^{2} / 4-$ $\arg \gamma+2 k \pi \mid \leq 2 \sin ^{-1}(\epsilon / 2 \sqrt{ } 2), k=0, \pm 1, \cdots$. Since the measure of $V$ is infinite, we can choose a set $S$, consisting of a finite number of the constituent intervals of $V$, of total measure exceeding $4 C$, whereupon the set $S_{\eta}=\sqrt{ } \eta S$ satisfies (6). Let us now pose the problem of maximizing the concentration on $S_{\eta}$ of a function $\Psi(u)$ which is the Fourier transform of a function $\psi(x)$ of unit norm supported on $|x| \leq 1$, i.e., let us seek to determine

$$
\max _{|| \downarrow * 1 \mid=1} \int_{s_{\eta}}|\Psi(u)|^{2} d u .
$$


The usual variation applied to this quadratic form shows that the maximum is given by the largest eigenvalue $\lambda_{1}$ of the Hermitian integral equation

$$
\frac{1}{(2 \pi)^{i / i}} \int_{-1}^{1} h_{\eta}(x-y) \psi(y) d y=\lambda \psi(x), \quad|x| \leq 1,
$$

in which the Fourier transform $H_{\eta}(u)$ of $h_{\eta}(x)$ is the characteristic function of the set $S_{\eta}$, i.e.,

$$
\begin{aligned}
H_{\eta}(u) & =1, & & u \in S_{\eta} \\
& =0, & & u \notin S_{\eta} .
\end{aligned}
$$

Moreover, the remaining eigenvalues of (8) measure the successively largest concentrations on $S_{\eta}$ attainable by the Fourier transforms of function of unit norm on $|x| \leq 1$ which are orthogonal to the eigenfunctions of (8) already determined. Thus if $N$ of the eigenvalues of (8) exceed $1-\epsilon^{2} / 8$, the corresponding eigenfunctions form a collection of $N$ mutually orthogonal $\epsilon$-approximate eigenfunctions of $A_{\eta}$ corresponding to the approximate eigenvalue $\gamma$. Since (8) is a Hermitian Hilbert-Schmidt operator with a continuous and positive definite kernel, we will be able to estimate $N$ in an easy way.

Let us denote the eigenvalues of $(8)$ by $\left\{\lambda_{k}(\eta)\right\}$ to stress their dependence on $\eta$. Since $\lambda_{k}(\eta)=\int_{S_{\eta}}\left|\Psi_{l_{i}}(u)\right|^{2}$ clu and

$$
0<\int_{s_{\eta}}\left|\Psi_{l_{i}}(u)\right|^{2} d u<\int_{-\infty}^{\infty}\left|\Psi_{k}(u)\right|^{2} d u=|| \psi_{k}||^{2}=1,
$$

we know that each $\lambda_{k}(\eta)$ necessarily lies between 0 and 1. By Mercer's theorem [2, p. 245], the sum of the eigenvalues is the trace of the kernel,

$$
\sum_{k=1}^{\infty} \lambda_{l i}(\eta)=\frac{1}{(2 \pi)^{1 / 2}} \int_{-1}^{1} h_{\eta}(0) d y=m\left(S_{\eta}\right) / \pi=m(S) \sqrt{ } \eta / \pi
$$

where $m(S)$ denotes the measure of $S$. We also have [2, p. 24:3]

$$
\sum_{k=1}^{\infty} \lambda_{k}{ }^{2}(\eta)=\frac{1}{2 \pi} \int_{-1}^{1} d y \int_{-1}^{1} d x\left|h_{\eta}(x-y)\right|^{2} .
$$

To estimate this integral, we apply the change of variables $y=p, x=p+\eta^{-1 / 2} t$, obtaining

$$
\eta^{-1 / 2} \sum_{k=1}^{\infty} \lambda_{k}{ }^{2}(\eta)=\frac{1}{2 \pi} \int_{-1}^{1} d p\left[\int_{V^{\prime} \eta(-1-\eta)}^{V^{\prime} \eta(1-p)} d t\left|\eta^{-1 / 2} h(t / \sqrt{ } \eta)\right|^{2}\right] .
$$

We now observe that $\eta^{-1 / 2} h(t / \sqrt{ } \eta)$ is the Fourier transform of the characteristic function of $S$, so that by (2)

$$
\int_{-\infty}^{\infty}\left|\eta^{-1 / 2} h(t / \sqrt{ } \eta)\right|^{2} d t=m(S)
$$

Thus the bracketed integrand is dominated by the constant $m(S)$, which of course is integrable on $|p| \leq 1$; moreover, for each $p,|p|<1$, this integrand approaches $m(S)$ as $\eta \rightarrow \infty$. Consequently by Lebesgue's dominated convergence theorem the right-hand side approaches $m(S) / \pi$. We conclude that

$$
\sum{\lambda_{k}}^{2}(\eta)=m(S) \sqrt{ } \eta / \pi-o(\sqrt{ } \eta)
$$


remarking parenthetically, for we make no use of that fact, that when $S$ is the union of a finite number of intervals, a slightly more accurate estimation of the integral shows the second-order term to behave like $K \log \eta$.

To describe the distribution of the eigenvalues, let $N_{\eta}(\theta)$, for $0<\theta<1$, denote the number of eigenvalues $\lambda_{k}(\eta)$ which are greater than $\theta$. The estimates (9) and (10) provide considerable information. To begin with, they show that the number of eigenvalues not close to 0 or 1 is $o(\sqrt{ } \eta)$. For by (9) and (10)

$$
J_{\eta}=\sum_{k} \lambda_{k}(\eta)\left[1-\lambda_{k}(\eta)\right]=\sum \lambda_{k}(\eta)-\sum \lambda_{k}^{2}(\eta)=o(\sqrt{ } \eta) .
$$

Now if $\alpha$ and $\beta$ are fixed, $0<\alpha<\beta<1$, each eigenvalue in the range

$$
\alpha<\lambda_{k}(\eta) \leq \beta
$$

contributes to $J_{\eta}$ an amount no smaller than $\tau=\min [\alpha(1-\alpha), \beta(1-\beta)]$. The number of such eigenvalues being $N_{\eta}(\alpha)-N_{\eta}(\beta)$, we find

$$
\tau\left[N_{\eta}(\alpha)-N_{\eta}(\beta)\right] \leq J_{\eta}=o(\sqrt{ } \eta)
$$

whence it follows that both $N_{+}=\lim \sup _{\eta \rightarrow \infty} \eta^{-1 / 2} N_{\eta}(\theta)$ and $N_{-}=\lim \inf _{\eta \rightarrow \infty} \eta^{-1 / 2} N_{\eta}(\theta)$ are independent of $\theta$ in the range $0<\theta<1$. To determine the latter quantities, we observe that

$$
\sum_{k} \lambda_{k}(\eta) \geq \theta N_{\eta}(\theta)
$$

and

$$
\begin{aligned}
\sum \lambda_{k}{ }^{2}(\eta) & =\sum_{k=1}^{x_{\eta}(\theta)} \lambda_{k}{ }^{2}(\eta)+\sum_{x_{\eta}(\theta)+1}^{\infty} \lambda_{k}{ }^{2}(\eta) \\
& \leq N_{\eta}(\theta)+\theta \sum_{N_{\eta}(\theta)+1}^{\infty} \lambda_{k}(\eta) \\
& \leq N_{\eta}(\theta)+\theta \sum_{k=1}^{\infty} \lambda_{k}(\eta) .
\end{aligned}
$$

Appealing again to (9) and (10) we find

$$
(1-\theta) m(S) / \pi \leq N_{-} \leq N_{+} \leq \theta^{-1} m(S) / \pi,
$$

and choosing $\theta$ near 1 on the right and near 0 on the left, we conclude that

$$
\lim _{\eta \rightarrow \infty} \eta^{-1 / 2} N_{\eta}(\theta)=m(S) / \pi
$$

for each $0<\theta<1$.

This is sufficient to establish the first assertion of the theorem. For given $\epsilon>0$, let $\theta=1-\epsilon^{2} / 8$. Since $m(S)>4 C$, (11) shows that for all $\eta$ sufficiently large there are at least $C \sqrt{ } \eta$ eigenfunctions $\left\{\psi_{k}\right\}$ of (8), orthonormal over $|x| \leq 1$, for which the Fourier transforms satisfy the concentration requirement (7) and which, in consequence, represent $\epsilon$-approximate eigenfunctions of $A_{\eta}$ corresponding to the approximate eigenvalue $\gamma$.

Finally, we observe that if $f=\sum_{1}{ }^{N} a_{k} \psi_{k}$, where $\left\{\psi_{k}\right\}$ are the eigenfunctions of (8) for which $\lambda_{k} \geq 1-\epsilon^{2} / 8$, then we find from (8) that, for the Fourier transform $F$ of $f$,

$$
\int_{s_{u}}|F(u)|^{2} d u=\sum\left|a_{k}\right|^{2} \lambda_{k} \geq\left(1-\epsilon^{2} / 8\right)|| f \|^{2} .
$$


Thus not only the functions $\left\{\psi_{k}\right\}$ themselves, but also any linear combination of them, will have Fourier transforms well-concentrated on $S_{\eta}$ and thus will serve as an $\epsilon$-approximate eigenfunction of $A_{\eta}$. Now if $\left|\gamma_{1}\right|=\left|\gamma_{2}\right|=1$, but $\gamma_{1} \neq \gamma_{2}$, let us take disjoint sets $S_{1}$ and $S_{2}$ satisfying (6) for $\gamma_{1}$ and $\gamma_{2}$ respectively, and construct, as above, two subspaces $\varepsilon_{1}$ and $\varepsilon_{2}$ of functions in $|x| \leq 1$ which have Fourier transform well-concentrated on $S_{1}$ and $S_{2}$, respectively. If $f_{1} \in \mathcal{E}_{1}$ and $f_{2} \in \mathcal{E}_{2}$, then $f_{1}$ and $f_{2}$ are not yet necessarily orthogonal, but they are nearly so, since their Fourier transforms are nearly concentrated on disjoint sets. Indeed, by Parseval's theorem,

$$
\begin{aligned}
& \int_{-1}^{1} f_{1}(x) \overline{f_{2}(x)} d x=\int_{-\infty}^{\infty} F_{1}(u) \overline{F_{2}(u)} d u \\
& =\int_{u s_{1}}+\int_{u\left(N_{1}\right.} \leq\left[\int_{s_{1}}\left|F_{1}(u)\right|^{2} d u \int_{s_{1}}\left|F_{2}(u)\right|^{2} d u\right]^{1 / 2} \\
& +\left[\int_{u \notin, s,}\left|F_{1}(u)\right|^{2} d u \int_{u \neq s, 1}\left|F_{2}(u)\right|^{2} d u\right]^{1 / 2} \\
& \leq 2 \epsilon / \sqrt{ } 8 \text {. }
\end{aligned}
$$

Thus $f_{2}$ can be projected into the orthogonal complement of $\varepsilon_{1}$ by a perturbation which is arbitrarily small, and which consequently will change neither the dimension of $\varepsilon_{2}$ nor the feature that $f_{2}$ was an approximate eigenfunction of $A_{\eta}$ corresponding to the approximate eigenvalue $\gamma_{2}$. It is in this way that $\varepsilon_{2}$ can be chosen to be orthogonal to $\varepsilon_{1}$. This completes the proof of the theorem.

\section{REFERENCES}

[1] J. A. Cochran and E. W. Hinds, Eigensystems associated with the complex-symmetric kernels of laser theory, SIAM J. Appl. Math. 26, 776-786 (1974)

[2] F. Riesz and B. Sz.-Nagy, Functional analysis, Ungar, N. Y., 1971

[3] H. J. Landau, On Szegö's cigenvalue distribution theorem and non-Hermitian kernels, J. d'Analyse Math. 28, 335-357 (1975)

[4] M. Kac, W. L. Murdock and G. Szegö, On the eigenvalues of certain Hermitian forms, J. Rat. Mech. Anal. 2, 767-800 (1953)

[5] E. G. Titchmarsh, Introduction to the theory of Fourier integrals, Oxford Univ. Press, 1948 